\title{
Mechanics of adhesive contacts: Experiment and theory
}

Cite as: AIP Conference Proceedings 2167, 020201 (2019); https://doi.org/10.1063/1.5132068

Published Online: 19 November 2019

lakov A. Lyashenko, and Valentin L. Popov

\section{ARTICLES YOU MAY BE INTERESTED IN}

Adhesive contribution to friction

AIP Conference Proceedings 2167, 020286 (2019); https://doi.org/10.1063/1.5132153

Regimes of adhesive wear in dry contact: Conditions of realization and determining parameters

AIP Conference Proceedings 2167, 020075 (2019); https://doi.org/10.1063/1.5131942

Active bio contact mechanics: Concepts of active control of wear and growth of the cartilage in natural joints

AIP Conference Proceedings 2167, 020285 (2019); https://doi.org/10.1063/1.5132152

\section{Lock-in Amplifiers up to $600 \mathrm{MHz}$}
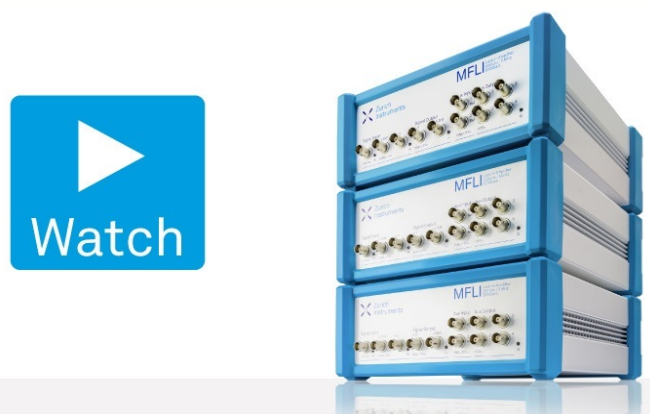


\title{
Mechanics of Adhesive Contacts: Experiment and Theory
}

\author{
Iakov A. Lyashenko ${ }^{1,2, a)}$ and Valentin L. Popov ${ }^{1,3,4, b)}$ \\ ${ }^{1}$ Technische Universität Berlin, Berlin, 10623 Germany \\ ${ }^{2}$ Sumy State University, Sumy, 40024 Ukraine \\ ${ }^{3}$ National Research Tomsk State University, Tomsk, 634050 Russia \\ ${ }^{4}$ National Research Tomsk Polytechnic University, Tomsk, 634050 Russia \\ a) i.liashenko@tu-berlin.de \\ b) Corresponding author: v.popov@tu-berlin.de
}

\begin{abstract}
Adhesive contacts between steel indenters and a layer of soft transparent rubber have been studied experimentally and numerically. Experiments were carried out using a setup allowing for slow exact positioning of the indenter in both normal and tangential direction and observing the contact area with a camera placed under the rubber layer. Indentation of flat-ended cylindrical punches showed that Boundary Element simulations very accurately describe experimental results. Changing velocity allowed determining the importance of viscoelastic effects on the adhesive contact.
\end{abstract}

Adhesion is both important and intriguing phenomenon in tribology. Despite studying of adhesion since many years, we are still far from complete understanding of this phenomenon. There are several classical theories and mathematical models of adhesion: the JKR theory [1] which is valid in the limiting case of very short-range adhesive interactions, DMT theory [2], applicable in the contrary limiting case of long-range adhesive interactions, as well as the theory by Maugis [3] considering arbitrary range of interactions, however, using a simplified interaction potential. The JKR and DMT theories are included in the Maugis theory as limiting cases. However, there are many problems, which wait for their solution. Thus, adhesion of rough surfaces is still discussed very controversially; the acceptable theory of adhesion of viscoelastic materials is practically absent. Even more complicated and controversial is adhesion in presence of tangential load, in particular the interrelation between adhesion and friction.

In [4], experimental equipment for investigation of flat-ended indenters during normal motion was designed and described. Authors developed numerical simulation procedure based on the boundary element method (BEM) and used it for simulation of adhesion of complex shaped indenters. They validated the results by comparison with experiments. In the present work, we improved the equipment, described in [4]. The main difference of our experimental setup is the presence of possibility of both normal and tangential motions. Extensive parameter studies with flat and rough surfaces, parabolic and cylindrical indenters, were carried out. One of the effects, which we were concentrated on, was the difference in force-displacement relations on the stages of indentation and detachment. This is very well-known effect, which even have been observed on the nanoscale - in AFM experiments [5]. Also, we investigated adhesion properties of flat indenters. Results of experiments are showed in Fig. 1.

In all experiments, cylindrical indenters with different radii were indented in the rubber layer to maximal indentation depth $d=0.4 \mathrm{~mm}$. Then indenters were pulled off to the moment of complete detachment. For each indenter, experiments were repeated 3 times, all three results are shown in Fig. 1. In the Fig. 1a, dashed lines show results, obtained in the framework of JKR theory in the half-space approximation. Figure shows rather good comparison between JKR theory and experiment only in the case of the indenter with diameter $4 \mathrm{~mm}$, because for half-space approximation diameter of indenter must be much smaller than thickness of the rubber. Solid lines represent the results obtained by BEM adapted for layered systems [6]. For simulation we used experimentally obtained parameters: elastic modulus of the rubber $E=0.324 \mathrm{MPa}$, surface energy $\gamma_{12}=0.326 \mathrm{~J} / \mathrm{m}^{2}$ and Poisson number $v=0.47$.

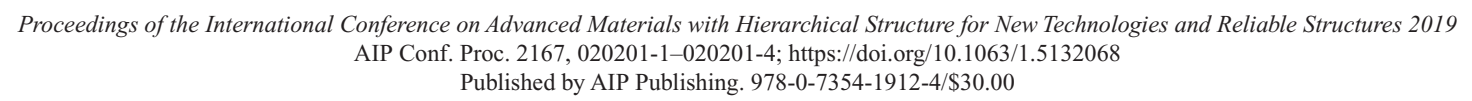




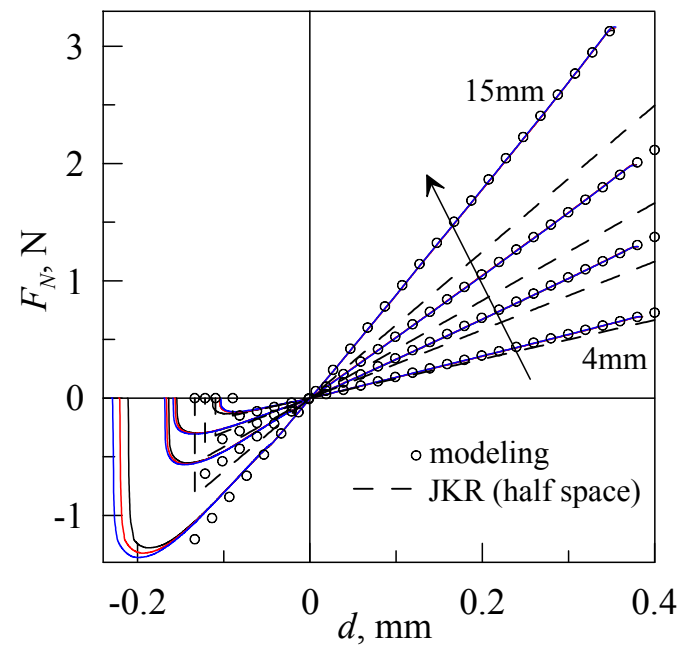

(a)

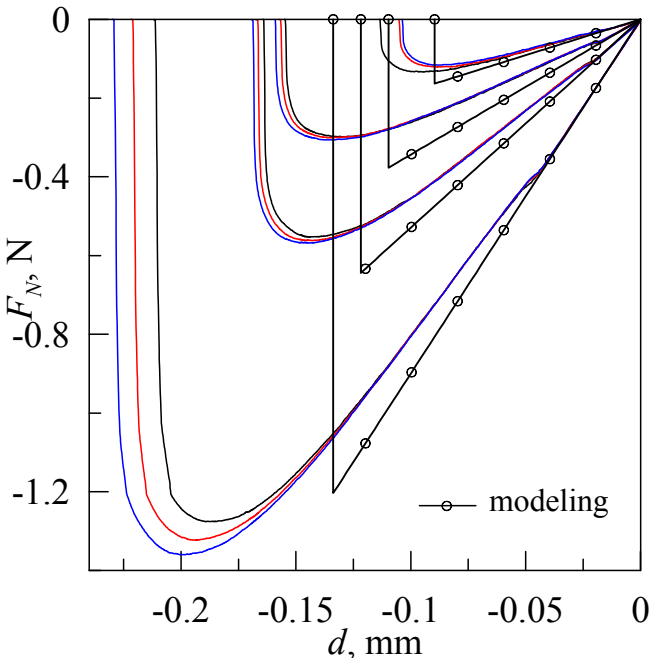

(b)

FIGURE 1. (a) Dependencies of normal force $F_{N}$ versus indentation depth $d$ for indentation of steel cylindrical indenters with diameters of 4, 7, 10 and $15 \mathrm{~mm}$ in a flat layer of rubber TARNAC CRG N3005 with thickness $h=25 \mathrm{~mm}$ (symbols). Dashed lines - theoretical predictions in the framework of JKR model for half-space approximation. Solid lines - simulation with BEM for layered systems; (b) Part of $F_{N}(d)$, depicted in Fig. 1a, in the area of detachment, without showing of JKR approximation

Rubber was located on the glass surface (in our simulations it was considered as a rigid material with infinitely large elastic modulus). In figure, one can see that the simulation results coincide with great accuracy only in the area of positive indentation depth. In region of negative forces (pulling stage), there is a relatively large difference (see Fig. 1b). First, in simulations we have sharp disappearance of the contact at one single critical displacement, but experimental results show rather slow detachment and several stable configurations of the contact (with area of the contact smaller than the radius of the cylinder).

Velocity of the indenter motion in the detachment area (Fig. 1b) was only $0.1 \mu \mathrm{m} / \mathrm{s}$, at this velocity the viscosity could not be the reason for this discrepancy. We are inclined to think that the reason is in the friction force in the boundary line of the contact. This fact must be investigated further, because in all experiments (both with flat and rough surfaces) we have observed the same behavior.

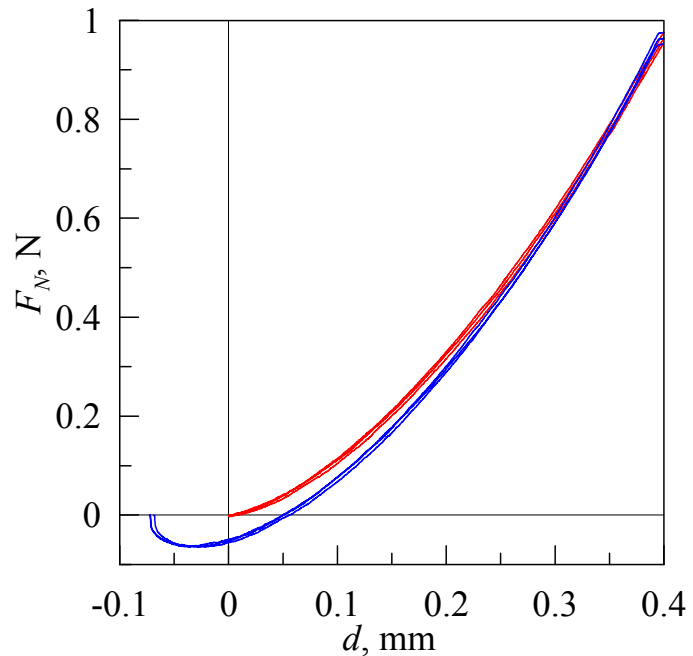

(a)

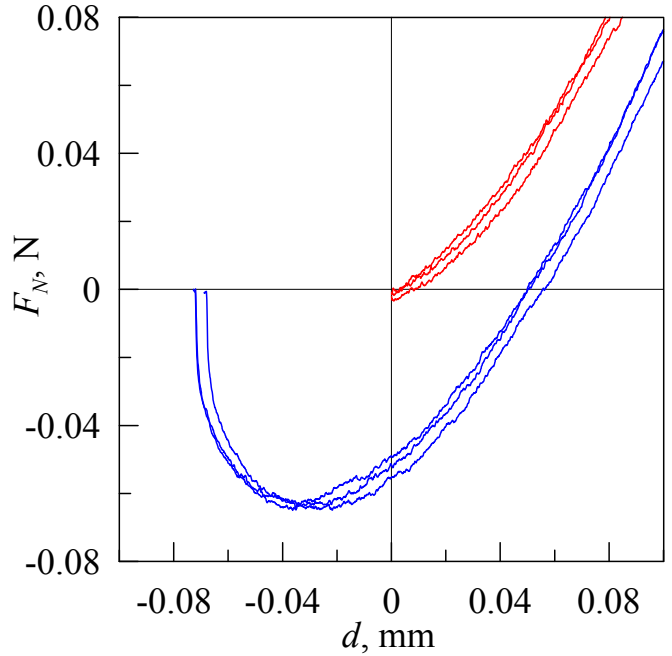

(b)

FIGURE 2. (a) Dependencies of the normal force $F_{N}$ versus indentation depth $d$ for indentation of steel spherical indenter with radius $R=33 \mathrm{~mm}$ in a flat layer of rubber TARNAC CRG N3005 with thickness $h=25 \mathrm{~mm}$. (b) Enlarged fragment of the figure 

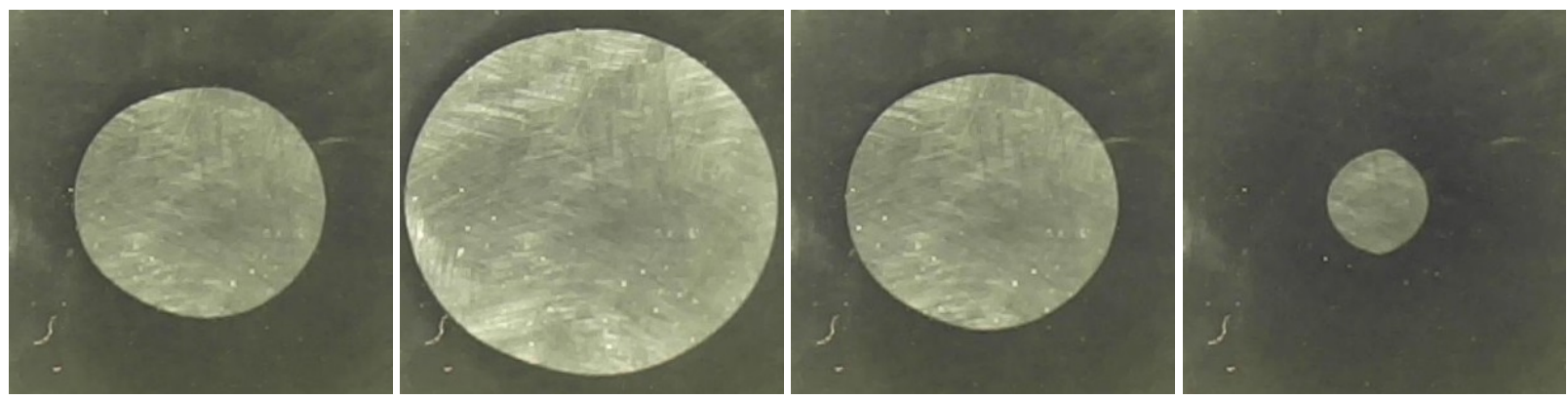

FIGURE 3. Images of contact area at indentation of steel spherical indenter with radius $R=33 \mathrm{~mm}$ in a flat layer of rubber TARNAC CRG N3005 with thickness $h=5 \mathrm{~mm}$. Images, located from left to right side, correspond to indentation depths $0.25 ; 0.5 ; 0.25$ and $0.0 \mathrm{~mm}$

For example, Figure 2 shows results of indentation of spherical indenter in the rubber material as we used for obtaining of results shown in Fig. 1. Here we also performed 3 experiments, and we can see good repeatability of results. At the stage of indentation, these dependencies started from at zero normal force, but during pulling off the normal force goes well below zero, because of adhesion interaction and the presence of adhesion neck. We found, that curves at indentation are well described by Hertz non adhesive contact model [7]. For the stage of detachment, much better approximation is given by the JKR model $[1,3]$.

Our experimental setup allowed for observing the changes in the contact area. A series of images of the contact area is presented in Fig. 3. The left image corresponds to indentation phase and indentation depth of $d=0.25 \mathrm{~mm}$. The second image from the left corresponds to the maximum value of indentation depth of $0.5 \mathrm{~mm}$. The third image corresponds again to $d=0.25 \mathrm{~mm}$, but during the phase of detachment. The last image was made at zero value of the indentation depth, clearly showing the existence of adhesive neck.

To determine the effect of viscoelasticity, we carried out experiments with different velocity of detachment. Figure 4a shows dependencies of normal force at indentation and detachment phases for a series of experiments with different detachment velocity.

The velocity of detachment was changed in a geometric progression from 1 to $128 \mu \mathrm{m} / \mathrm{s}$. Particular values were: $1,2,4,8,16,32,64$ and $128 \mu \mathrm{m} / \mathrm{s}$. Figire $4 \mathrm{~b}$ shows dependence of the absolute value of the negative minimum normal force (which usually is identified as "force of adhesion") versus velocity of motion of indenter. From figure it follows, that minimum $F$ significantly increased for last three values of the velocity. This effect is due to viscoelasticity of rubber layer [8]. At big values of velocity, we observe bigger values of normal forces during detachment of indenter; for indenters with bigger radii this effect becomes more pronounced.

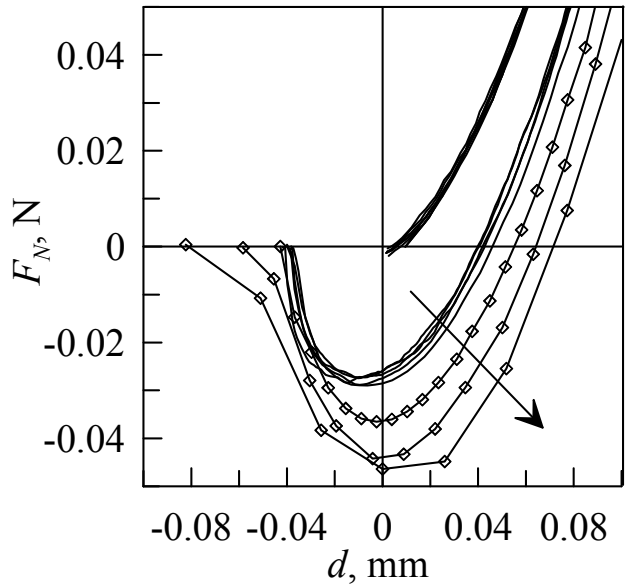

(a)

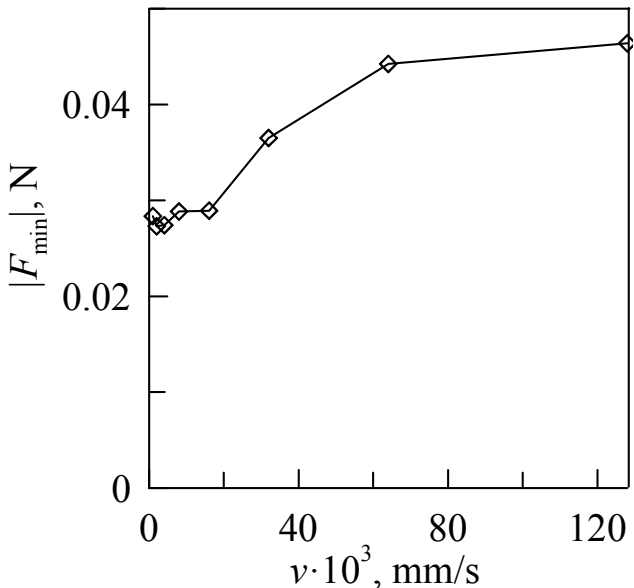

(b)

FIGURE 4. (a) Dependencies of the normal force $F_{N}$ versus indentation depth $d$ for indentation of a steel spherical indenter with radius $R=33 \mathrm{~mm}$ in a flat layer of rubber TARNAC CRG N3005 with thickness $h=5 \mathrm{~mm}$;

(b) dependence of the minimum normal force (absolute value) versus velocity of motion $v$ 


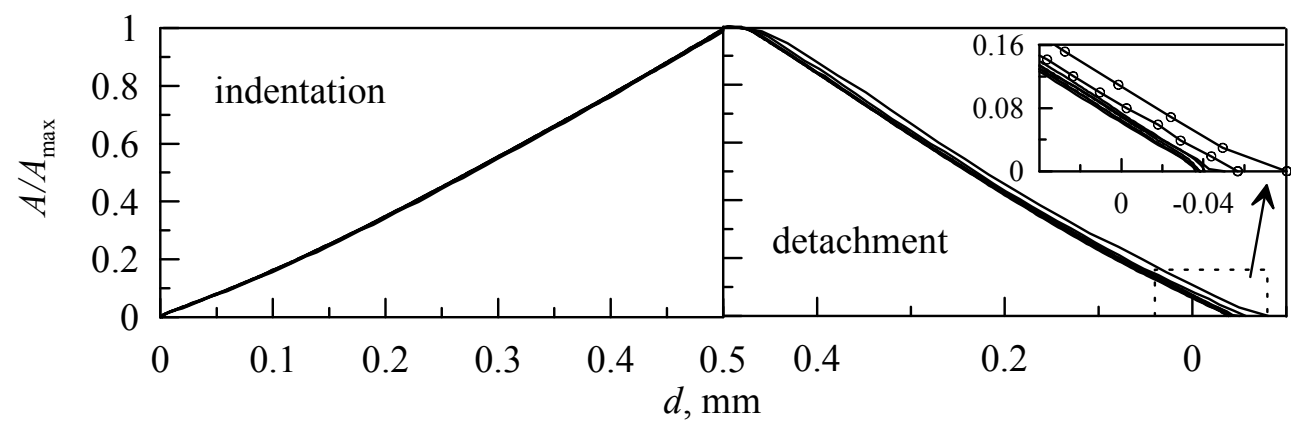

FIGURE 5. Dependencies of normalized contact area versus indentation depth $d$, correspond to dependencies, shown in Fig. 4a

Dependencies of the normalized contact area $A / A_{\max }$ versus indentation depth $d$ for both phases of motion, indentation and detachment (motions in two opposite directions), are presented in Fig. 5. During the indentation phase, the force- displacement relation is well described by the Hertz theory. We thus await that this will also be the case for the area dependency. According to Hertz, the contact radius is equal $a=\sqrt{R d}$, in our case indenter radius $R=33 \mathrm{~mm}$ and the contact area $A=\pi a^{2}$, to $A=\pi R d$. Thus, the dependence $A / A_{\max }(d)$ should be linear. Note, that due to shape defects, the contact had a small ellipticity (see Fig. 3). This may be a reason for a moderate deviation from straight line of the dependency in left panel of Fig. 5.

In Fig. 5 showed dependencies of the normal force. Note that the dependency of the contact area on the indentation depth has much weaker velocity dependency compared with that of the normal force. Observation that is even more striking is that the contact area vanishes continuously, while according to the JKR theory the detachment should be jump like. The reason for this continuity is not clarified yet. One reason might be the roughness. Indeed, numerical simulation with BEM carried out in [9] illustrated that the jump can disappear due to roughness of surfaces. Results in Figs. 5 and 4 show that at increasing of velocity of motion contact detach at bigger absolute values of negative indentation depth, due to presence of viscoelastic effects in the contact. However, simulations in [9] were carried out for elastic bodies. Technique proposed recently in [10] could be used for analyzing rough viscoelastic contacts. Our experiments show that there are still many fundamental question in the theory of adhesion, which wait for their solution. In near future, we plan to investigate in detail adhesion of rough surfaces, based on developed experimental and numerical techniques.

\section{ACKNOWLEDGMENTS}

Authors acknowledge financial support of the Deutsche Forschungsgemeinschaft (DFG PO 810-55-1).

\section{REFERENCES}

1. K. L. Johnson, K. Kendall and A. D. Roberts, Proc. Royal Soc. Lond. A 324, 301-313 (1971).

2. B. V. Derjaguin, V. M. Muller and Yu. P. Toporov, J. Colloid Interface Sci. 53, 314-326 (1975).

3. D. Maugis, J. Colloid Interface Sci. 150, 243-269 (1992).

4. V. L. Popov, R. Pohrt, and Q. Li, Friction 5, 308-325 (2017).

5. R. Buzio and U. Valbusa, J. Phys. Condens. Matter 20, 354014:1-9 (2008).

6. Q. Li, R. Pohrt, I. A. Lyashenko, and V. L. Popov, Proc. Inst. Mech. Eng. Part J.-J. Eng. Tribol., doi $10.1177 / 1350650119854250$ (2019).

7. H. Hertz, Journal für die reine und angewandte Mathematik 92, 156-171 (1882).

8. Z. Liu, H. Lu, Y. Zheng, D. Tao, Y. Meng, and Y. Tian, Sci. Reports 8, 6147:1-8 (2018).

9. Q. Li, R. Pohrt, and V.L. Popov, Front. Mech. Eng. 5, 7 (2019).

10. J. S. van Dokkum and L. Nicola, Modelling Simul. Mater. Sci. Eng., doi 10.1088/1361-651X/ab3031 (2019). 